\title{
Characterization of the blob generation region and blobby transport in a stellarator
}

\author{
G. Fuchert ${ }^{1}$, G. Birkenmeier ${ }^{1,2}$, M. Ramisch ${ }^{3}$, U. Stroth ${ }^{1,2}$ \\ ${ }^{1}$ Max-Planck-Institut für Plasmaphysik, Boltzmannstr. 2, 85748 Garching, Germany \\ 2 Physik-Department E28, Technische Universität München, James-Franck-Str. 1, \\ 85748 Garching, Germany \\ ${ }^{3}$ Institut für Grenzflächenverfahrenstechnik und Plasmatechnologie, Universität \\ Stuttgart, 70569 Stuttgart, Germany \\ E-mail: golo.fuchert@ipp.mpg.de
}

\begin{abstract}
Filaments of increased pressure ("blobs") in the scrape-off layer of toroidally confined magnetized plasmas are studied in the context of fusion research due to their relevance for confinement and wall safety. Analytical models in simple toroidal magnetic field geometries have proven useful to get a quantitative understanding of blob dynamics in tokamaks. However, their direct applicability to the more complicated stellarator geometry is far less studied. The experiments presented here show that in the stellarator TJ-K blobs are field-aligned structures occurring in scrape-off layer regions of negative mean normal curvature, which is in agreement with common blob models. Furthermore, it is shown that in TJ-K, in accordance with findings from tokamaks, blobs account for a significant fraction of the turbulent scrape-off layer transport of the order of several tens of percent.
\end{abstract}

PACS numbers: 52.25.Xz,52.30.-q,52.35.Ra,52.55.Hc,52.70.Kz 


\section{Introduction}

Turbulence generated filaments of increased pressure ("blobs" [1]) are commonly found in the scrape-off layer (SOL) of toroidally confined magnetized plasmas. They are observed to be aligned to the magnetic field $\mathbf{B}[2-4]$ and to propagate radially outward [5], transporting energy and particles. They are actively studied due to their relevance for SOL transport and wall safety in fusion experiments.

In the poloidal direction, blobs mainly follow the background $\mathbf{E} \times \mathbf{B}$ drift combined with magnetic shear effects due to the field alignment $[6,7]$. In contrast, the observed radial propagation is a characteristic feature of the blobs. Basic analytical blob models [8-10] in simple toroidal magnetic field geometry explain this propagation as follows: Due to charge-separating drifts the blob is polarized in the poloidal direction. The resulting electrical field perturbation $\tilde{\mathbf{E}}$ then leads to $\tilde{\mathbf{E}} \times \mathbf{B}$ drifts in the background magnetic field $\mathbf{B}$. These drifts point dominantly into the radial direction and feature drift velocities of up to ten percent of the ion sound speed $c_{\mathrm{s}}$ [11]. In toroidal magnetic field geometry the charge separating drifts are dominantly caused by curvature effects $[11,12]$. The resulting radial drift velocity $v_{\mathrm{r}, \mathrm{b}}$ is typically estimated from quasineutrality $(\nabla \cdot \mathbf{j}=0)$, leading to a vorticity equation. Predictions of these analytical models typically describe many features of blob dynamics observed in the experiment, although they typically neglect interactions with the ambient turbulence and assume that the background plasma profiles remain fixed. For TJ-K it was shown that the relevant contributions to the charge balance are the curvature-induced currents, the ion-polarization current and parallel sheath currents [13]. In this case the vorticity equation can be written as [14]

$$
\frac{m_{i}}{B^{2}} \nabla \cdot\left(n \frac{\mathrm{d}}{\mathrm{d} t} \nabla_{\perp} \phi\right)=\nabla_{\|} j_{\|}+\frac{2}{B} \hat{\mathbf{b}} \cdot \boldsymbol{\kappa} \times \nabla p
$$

with $\mathrm{d} / \mathrm{d} t=\left(\partial / \partial t+\mathbf{v}_{E \times B} \cdot \nabla\right)$, the plasma potential $\phi$, the ion mass $m_{i}$, the plasma density $n$, the unit vector in the direction of the magnetic field $\hat{\mathbf{b}}=\mathbf{B} / B$, the curvature vector $\boldsymbol{\kappa}=(\hat{\mathbf{b}} \cdot \nabla) \hat{\mathbf{b}}$, and the plasma pressure $p$. Applying a series of assumptions, Eq. (1) or similar model equations are used to find scaling laws for the radial velocity of blobs (see e. g. Refs. [8-10, 14, 15] or more recently including warm ion effects Ref. [16]).

It turns out that, in analogy to the interchange instability, the drive for the radial propagation of blobs relies on a negative normal curvature $\kappa_{\mathrm{n}}=\boldsymbol{\kappa} \cdot \hat{n}$ ( $\hat{n}$ is the normal vector of the flux surfaces) and is, hence, often referred to as interchange drive. Due to the interchange drive it can be expected that blobs are only generated in regions where $\kappa_{\mathrm{n}}$ is negative, where as a result a larger turbulent SOL transport should be observed.

For the derivation of scaling laws for the radial blob velocity, Eq. (1) (or comparable model equations) is often treated in slab geometry, where $x$ represents the radial direction, $y$ the poloidal one, and $z$ the direction of the magnetic field. By assuming $\boldsymbol{\kappa}=-1 / R \cdot \hat{\boldsymbol{e}}_{x}$ (i. e. $\kappa_{\mathrm{n}}=-1 / R$ at the outer midplane) and $\boldsymbol{B}=B \hat{\boldsymbol{e}}_{z}$ (with the unit 


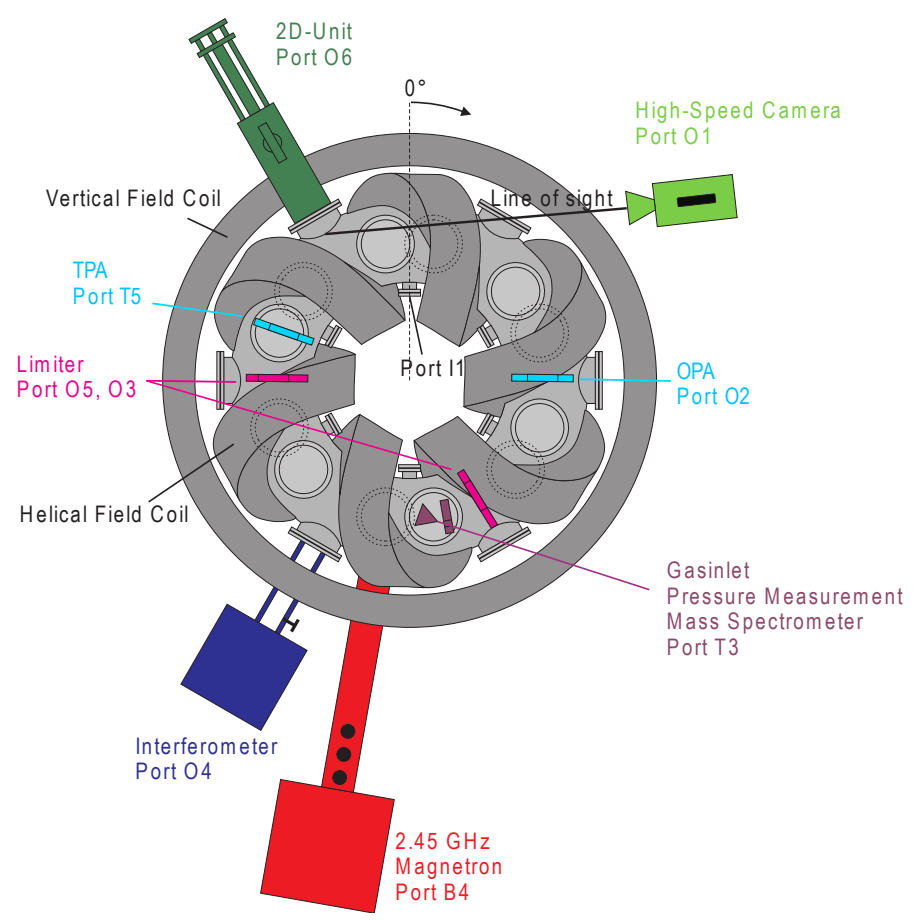

Figure 1. Experimental setup at TJ-K with two limiter plates located at ports O3 and O5. The main diagnostics for the presented discharges were two poloidal probe arrays (at ports $\mathrm{O} 2$ and $\mathrm{T} 5$ ) and a fast camera observing the plasma tangentially at O6.

vectors $\hat{e}_{x, y}$ the drive term can be approximated as $[12,14]$

$$
\frac{2}{B} \hat{\mathbf{b}} \cdot \boldsymbol{\kappa} \times \nabla p \approx-\frac{2 c_{\mathrm{s}}^{2}}{R} \frac{m_{i}}{B} \frac{\partial n}{\partial y} .
$$

While this leads to scaling laws which are able to predict the radial velocity of blobs in tokamaks (see Ref. [11] for a relatively recent summary of experimental investigations) it cannot be expected to describe blob dynamics in the complex magnetic field geometry of stellarators directly, where $\kappa=-1 / R \cdot \hat{\boldsymbol{e}}_{x}$ is not a good assumption. Although the existence of blobs in stellarators is clearly documented for many different fusion experiments (e.g. HSX [17], LHD [18], TJ-K [19], TJ-II [20], WEGA [21], and W7AS [22]), there are almost no systematic comparisons of the measured velocities with predictions from the analytical scaling laws (see Ref. [13] for such a comparison in TJ-K, where a good agreement was found) or predictions of the basic blob model in general.

In this paper experiments conducted in the stellarator TJ-K, introduced in Sec. 2, are presented, which study the role of the normal curvature on the blob generation (Sec. 3) and the relevance of blobs for the turbulent transport (Sec. 4). Final conclusions and a summary of the results are given in Sec. 5 . 

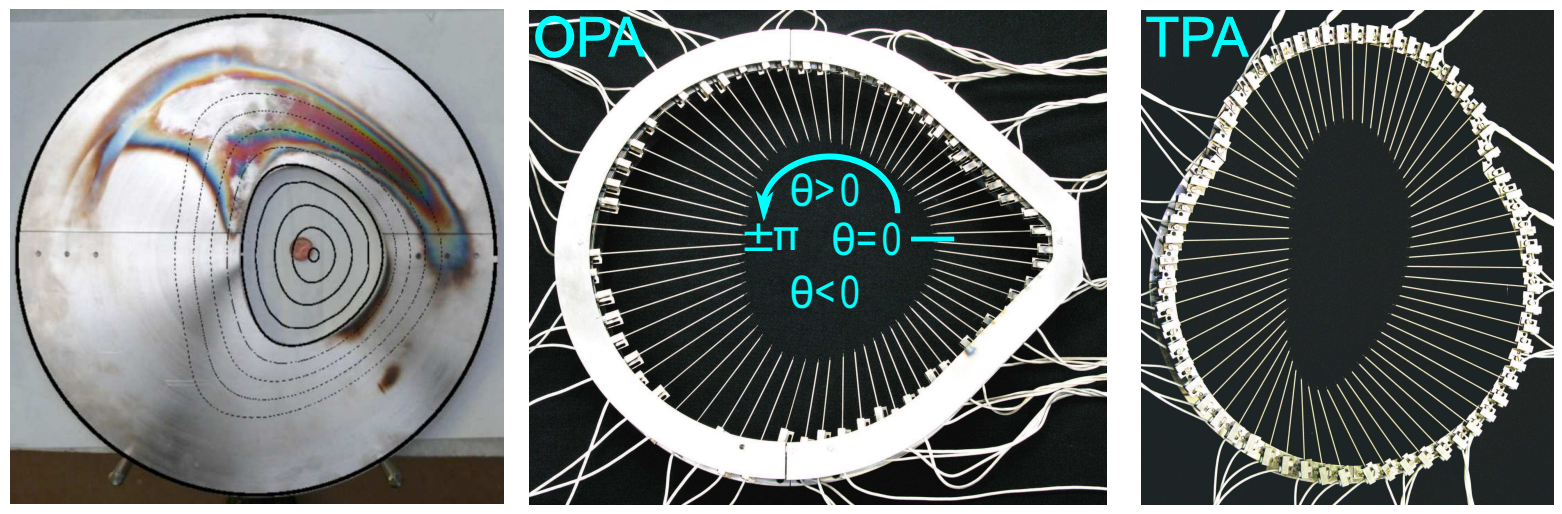

Figure 2. Photograph of a poloidal limiter disk (left), overlayed with a poloidal cross section of closed flux surfaces (solid lines) in the confined plasma and flux surfaces of the standard TJ-K setup now intersected by the disk (dashed lines), together with photographs of the outer port array (OPA) and the top port array (TPA). Both poloidal probe arrays feature 64 probes aligned to the same flux surface at the respective toroidal measurement positions. The outboard side is to the right $(\theta=0)$.

\section{Experimental setup}

The presented measurements were performed in helium and hydrogen discharges in the stellarator TJ-K [23, 24] (see Fig. 1) with a magnetic field strength of $70 \mathrm{mT}$, heated with microwaves at $2.45 \mathrm{GHz}$. In this discharge scenario, the typical electron density $n_{e}$ is below $10^{18} \mathrm{~m}^{-3}$ in the core and $10^{17} \mathrm{~m}^{-3}$ in the SOL, while the electron temperature $T_{e}$ is between 5 and $10 \mathrm{eV}$ throughout the whole plasma. The plasma was limited by two poloidal limiter disks (Fig. 2), which were introduced to enlarge the scrape-off layer (SOL) with constant connections lengths (see Fig. 1 for the employed setup at TJ-K). The limiters are electrically connected to the walls. The blob structure and SOL transport were studied with two poloidal Langmuir probe arrays and a high-speed camera. The discharges analyzed in this paper are comparable to those analyzed in Ref. [13]. Hence, general information about blob dynamics in this discharge scenario of TJ-K, including size and velocity scalings, can be found in that reference.

The 64 probes on each of the probe arrays are aligned to the same flux surface at an effective radius of $r_{\text {eff }} \approx 6.5 \mathrm{~cm}$. Due to the limiters, however, the effective radius of the last closed flux-surface (typically located at $r_{\text {eff }} \approx 9 \mathrm{~cm}$ ) is $r_{\text {eff }} \approx 3 \mathrm{~cm}$ and, hence, the probes are located in the SOL defined by the limiters. The difference of about $6 \mathrm{~cm}$ is the width of the SOL region with constant connection lengths, as it is assumed in analytical blob models. In TJ-K, blobs are observed to propagate across the full radial width of this region $[13,19]$.

Figure 2 shows a photograph of the poloidal probe arrays and introduces the poloidal angle $\theta \in[-\pi, \pi]$ (with 0 at the outboard side) used throughout this paper. The outer port array (OPA) is suited for measurements in the triangular plane at the six outer ports of TJ-K (six-fold symmetry of the magnetic field) and the top port array (TPA) at the six top ports. Depending on the probe setup, both arrays can 
be employed to measure either fluctuations in the density or potential or the radial turbulent transport. A detailed description of the probe arrays and their measurement capabilities can be found in Refs. [25, 26].

The high-speed camera is a Photron Fastcam SA-5, it observes the plasma tangentially at the outer port position O6. Intensity fluctuations in the image data represent density fluctuations in the plasma. Shown throughout this paper are normalized intensity fluctuations $\tilde{I}_{n}=(I-\langle I\rangle) /\langle I\rangle$. The interpretation of the image data in the stellarator geometry of TJ-K with special focus on blob identification is discussed in Ref. [13].

All probe data is gathered simultaneous for $1 \mathrm{~s}$ at an acquisition rate of $1 \mathrm{MHz}$. For combined measurements of the probe arrays and the fast camera an acquisition rate of $0.15 \mathrm{MHz}$ is used to match the frame rate of the camera.

For the data analysis of both diagnostics, the conditional averaging and cross correlation techniques are used. A summary of both techniques specifically for analyzing blobs in the scrape-off layer can be found in Ref. [22].

From the interchange drive of blobs, as explained in the introduction, one could imagine that for field-aligned, elongated structures the field-line averaged normal curvature $\left\langle\kappa_{\mathrm{n}}\right\rangle$ is the relevant quantity to be examined rather than the local values of $\kappa_{\mathrm{n}}$. When the segment of a field line with connection length $l_{\mathrm{c}}$ between the two limiter disks is parametrized as $\mathbf{s}(t),\left\langle\kappa_{\mathrm{n}}\right\rangle$ can be calculated using a magnetic field line tracing code by numerically evaluating

$$
\left\langle\kappa_{\mathrm{n}}\right\rangle \approx \frac{1}{l_{\mathrm{c}}} \int_{0}^{l_{\mathrm{c}}} \kappa_{\mathrm{n}}(\mathbf{s}(t)) \mathrm{d} t .
$$

\section{Blob region in TJ-K}

The factor $1 / R$ in Eq. (2) represents a constant normal curvature. Since in stellarators $\kappa_{\mathrm{n}}$ varies strongly along field lines, it is more accurate to replace $1 / R$ by the field-line averaged normal curvature $\left\langle\kappa_{\mathrm{n}}\right\rangle$. In the common picture of interchange driven blob dynamics, blobs should only be generated in "bad curvature" regions where $\left\langle\kappa_{\mathrm{n}}\right\rangle<0$.

The usage of field-line averaged quantities (averaged between the limiter plates) to describe the blob dynamics requires that the filaments are indeed fully extended between both limiters. This was checked by correlating the probes of one poloidal array with the field-line connected probes on the other probe array. The result is shown in Fig. 3 as a function of the poloidal angle of the reference OPA probe (refer to Fig. 2 for the introduction of the probe arrays and the poloidal coordinate $\theta$ ). The region of negative $\left\langle\kappa_{\mathrm{n}}\right\rangle$ is shown in the plot as gray shaded area. Three different regions can be distinguished in the plot: For OPA probes with poloidal angles $\theta \lesssim-0.45 \pi$ (region I) the cross correlation values are small $(<0.5)$ compared to larger angles. Largest correlation values (mostly $>0.5$ ) are found in the region $-0.45 \pi \lesssim \theta \lesssim 0.65 \pi$ (region II), where $\left\langle\kappa_{\mathrm{n}}\right\rangle$ is negative. For $\theta \gtrsim 0.65 \pi$ (region III) $\left\langle\kappa_{\mathrm{n}}\right\rangle$ is positive and the cross correlation values are still large but slowly decreasing. The large cross correlation values indicate 


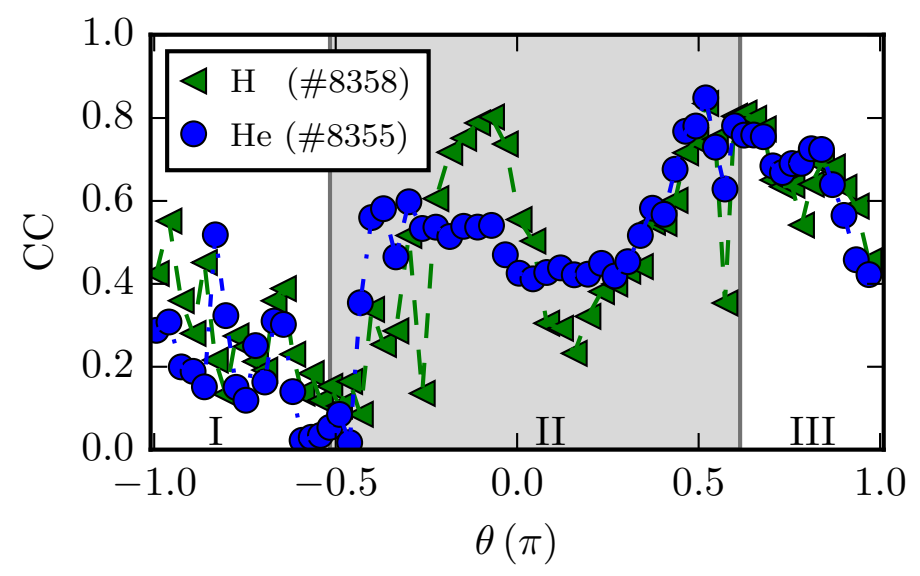

Figure 3. Cross correlation (at $\tau=0$ ) of the ion saturation current signals of reference probes on the OPA with their field-line connected probes on the TPA as a function of the poloidal angle of the OPA reference probe. Regions of negative $\left\langle\kappa_{n}\right\rangle$ are shown in gray.

the presence of structures extended along field lines between the two probe arrays. It will be shown in the following that this result is caused by blobs which are absent in region I, generated in region II and propagate poloidally into region III. In TJ-K, blobs in the SOL propagate poloidally in the ion diamagnetic direction, mainly due to the background $\mathbf{E} \times \mathbf{B}$ rotation [19]. Note that prior studies in TJ-K [13, 19] report blob generation by drift-wave turbulence in the vicinity of the LCFS at poloidal locations included in region II.

To show that the large cross correlation values are caused by blobs, the cross correlation is calculated with the same reference probes on the OPA, but this time with the different pixels of the image data acquired by the fast camera. The result is shown in Fig. 4 for different reference probes, one from each region described above (within the different regions, the qualitative result does not depend on the choice of the reference probe). The displayed images correspond to the $2 \mathrm{D}$ cross correlation functions at the time lag $\tau_{\max }$ where the highest cross correlation value is found between the respective reference probe and the different camera pixels. For $\theta=-0.54 \pi$ (region I), Fig. 4 a), no significant correlation is found between probe and image data. For $\theta=-0.35 \pi$ (region II), Fig. $4 \mathrm{~b}$ ), a blob in the SOL is seen in the cross correlation data at $\tau_{\max }=40 \mu \mathrm{s}$. The positive time lag means that the structure appears in the field of view of the camera after it reached the OPA reference probe. For the third reference probe at $\theta=0.70 \pi$ (region III), Fig. 4 c), a blob is observed reaching the highest cross correlation values at the same location as for the reference probe in region II but at $\tau_{\max }=-100 \mu \mathrm{s}$, meaning that this time it is visible in the image data before it reaches the reference probe. It can be concluded that the high cross correlation values in Fig. 3 are caused by blobs in the SOL with a parallel length $l_{\|}$larger than the distance between the two probe arrays $\left(l_{\mathrm{OPA} \rightarrow \mathrm{TPA}} \approx 1.85 \mathrm{~m}\right)$. Since this value is close to the connection length between both limiters $\left(l_{\mathrm{SOL}} \approx 2.79 \mathrm{~m}\right)$ it is assumed that indeed $l_{\|}=l_{\mathrm{SOL}}$. By introducing additional 
reference probes $1 \mathrm{~cm}$ in front of the limiter discs it was confirmed at least locally that the filaments in the SOL of TJ-K indeed cover the full connection length.

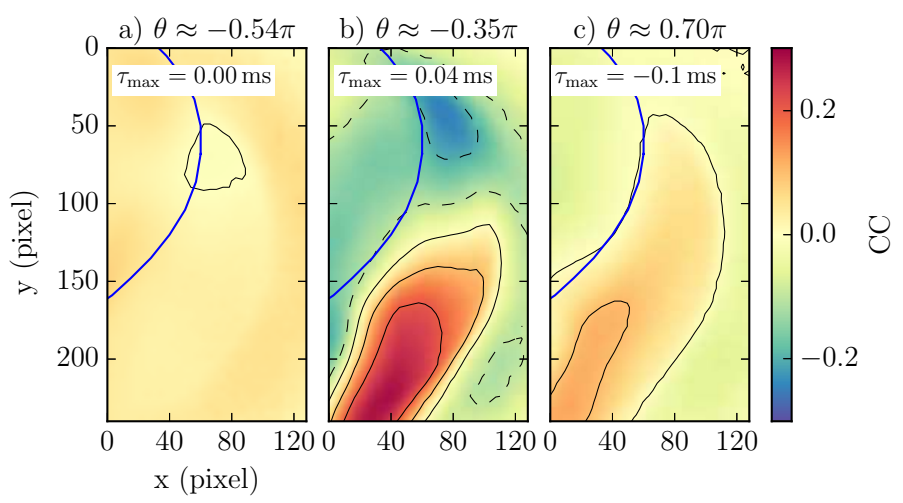

Figure 4. Cross correlation (CC) of the image data (in image coordinates $x, z$ ) with three OPA probes at different poloidal angles $\theta$ (stated above the images). For each reference probe, the image of time lag $\tau_{\max }$ (time where the highest value in the $\mathrm{CC}$ occurs) is shown. The projection of the last closed flux-surface (LCFS) on the camera data is shown as blue line, the SOL is right of the LCFS.

The cross correlation analysis presented above revealed that blobs are observed in a specific region of the SOL and that the poloidal propagation has an influence on the poloidal extent of this region. In the following the role of the poloidal propagation is studied further to determine the SOL region where blobs are generated.

After a blob is generated it propagates radially outwards and is detected as soon as it reaches a probe on one of the probe arrays. Due to the poloidal propagation, the same blob is detected by other poloidally separated probes of the same array at a later time. This can be visualized in a $2 \mathrm{D}$ plot by calculating the cross correlation functions of one reference probe with all other probes on the same array and plotting it as a function of the poloidal probe position and time. In this representation, the poloidal blob propagation is seen as stripe-like pattern.

A comparison of the cross correlation functions for different reference probe positions on the OPA as well as the TPA is shown in Fig. 5. In the top row, the results for the OPA are shown, where in a) the reference probe is located at the inboard side and in b) at the outboard side. The same situation is shown in the bottom row for the TPA, again for a reference probe at the inboard side in c) and one on the outboard side in d). For both probe arrays no blobs are detected by the reference probe on the inboard side, only at the outboard side. The poloidal range in which blobs are observed (appearance of the stripe pattern in the cross correlation) by the respective probe array, in the following called blob region, is marked by the horizontal black lines in Fig. 5. The blob region corresponds roughly with the regions II and III discussed above. Note that the periodicity seen on time scales of about $100 \mu \mathrm{s}$ is caused by multiple blob generation by quasi-coherent modes arising from drift-wave turbulence in the confined plasma, as was discussed in Ref [13]. 
Since the poloidal propagation direction is reversed together with the magnetic field direction [26], a similar analysis has been performed for reversed magnetic field and the result is shown in Fig. 6. The blob region (again shown by the horizontal black lines) is observed at different poloidal angles compared to the standard field case, as can clearly be seen by the fact that the cross correlation analysis now shows blobs for all four reference probes in contrast to the standard field case shown in Fig. 5. The reason for this observation will become clear in the following.

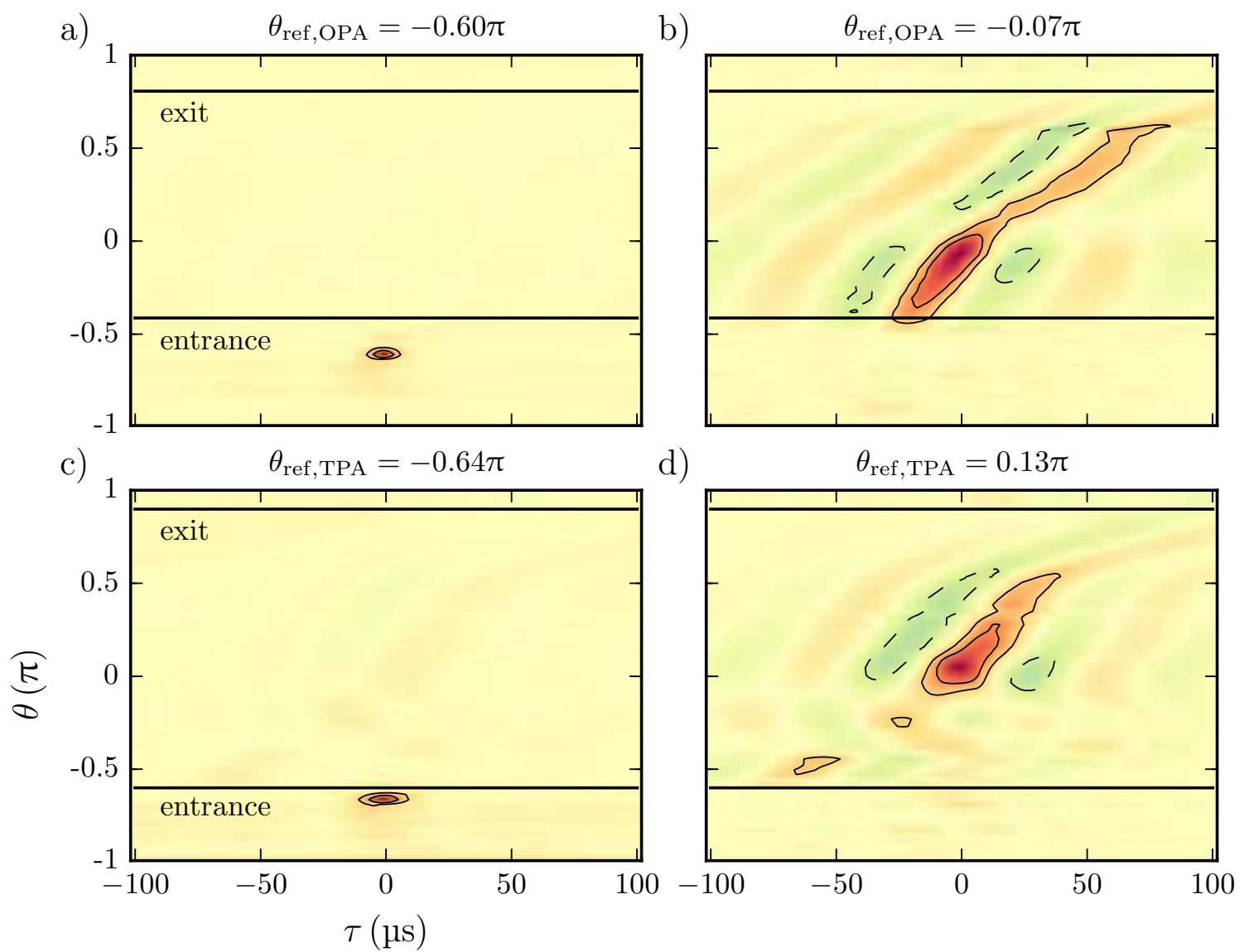

Figure 5. Cross correlations for different reference probes on the OPA (top row) and TPA (bottom row) with all other probes on the same probe array (\#8355). Positive correlation values are shown in red with closed contour lines, negative ones in blue with dashed contour lines. Stripe-like patterns point to the existence of blobs, which are observed within the range marked by the horizontal black lines.

It is useful to define the entrance and exit of the blob region. The entrance is located at the poloidal position the blobs move poloidally away from and the exit is the position the blobs move towards (see Figs. 5 and 6). In the standard magnetic field case, the entrance is located at negative poloidal angles and in the reversed field case at positive ones. Field line tracing revealed that for both magnetic field directions the entrance angles observed for the OPA and the TPA are connected via a field line. This is not the case, however, for the exit positions of the blob region, which may indicate that filaments close to the exit positions are no longer perfectly aligned to the field lines. 


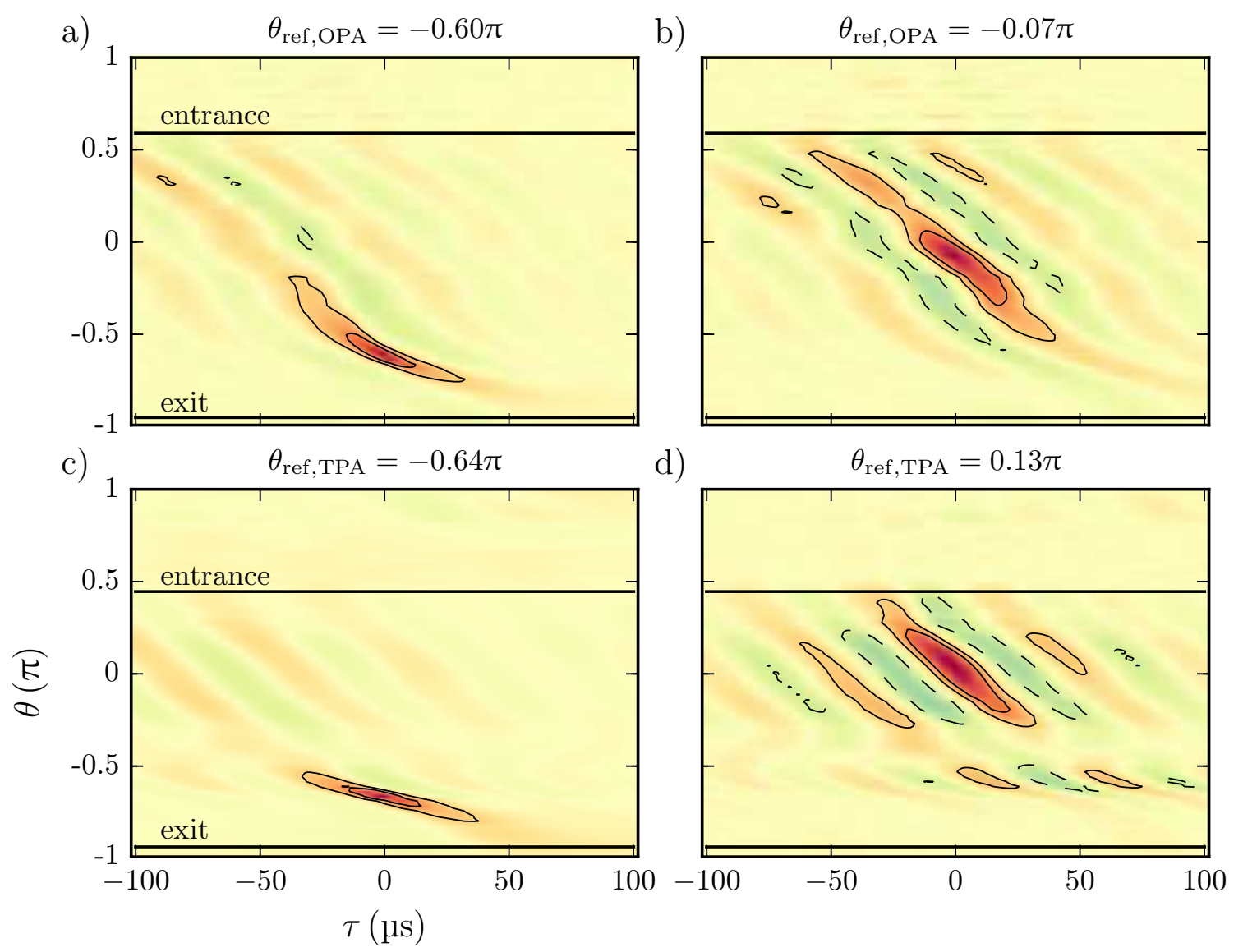

Figure 6. Same representation as in Fig. 5, but for reversed magnetic field.

It can be concluded that the region in the SOL of TJ-K where blobs are observed, is a poloidally extended band that twists toroidally around the plasma, following the helical magnetic field lines. Hence, concerning blob dynamics, the limiter disks break the stellarator symmetry in the sense that blobs are observed at different poloidal positions depending on the toroidal angle at which measurements are performed (in this case the locations of the OPA and TPA).

In order to study the connection between the blob region and the normal curvature, both the local $\kappa_{\mathrm{n}}$ and the averaged $\left\langle\kappa_{\mathrm{n}}\right\rangle$ are shown as a function of the poloidal angle in Fig. 7 a) at the toroidal position of the OPA and in b) at the position of the TPA. It can be seen that for both probe arrays neither the region of negative $\kappa_{\mathrm{n}}$ nor $\left\langle\kappa_{\mathrm{n}}\right\rangle$ completely matches the blob region identified in the last section (gray shaded area).

The poloidal propagation direction is marked in the figures by the black arrow. For both probe arrays the entrance of the blob region (left edge of the gray area at low poloidal angles $\theta$ ) is located close to the zero crossing of $\left\langle\kappa_{\mathrm{n}}\right\rangle$, while the local value of $\kappa_{\mathrm{n}}$ is positive for the TPA and negative for the OPA. The exit of the blob region is located in regions where both $\left\langle\kappa_{\mathrm{n}}\right\rangle$ and $\kappa_{\mathrm{n}}$ are positive (i.e. in an interchange stable region). A similar analysis for the reversed field case is shown in Fig. 8. While the normal curvature is unaffected by the field reversal, the poloidal propagation direction 

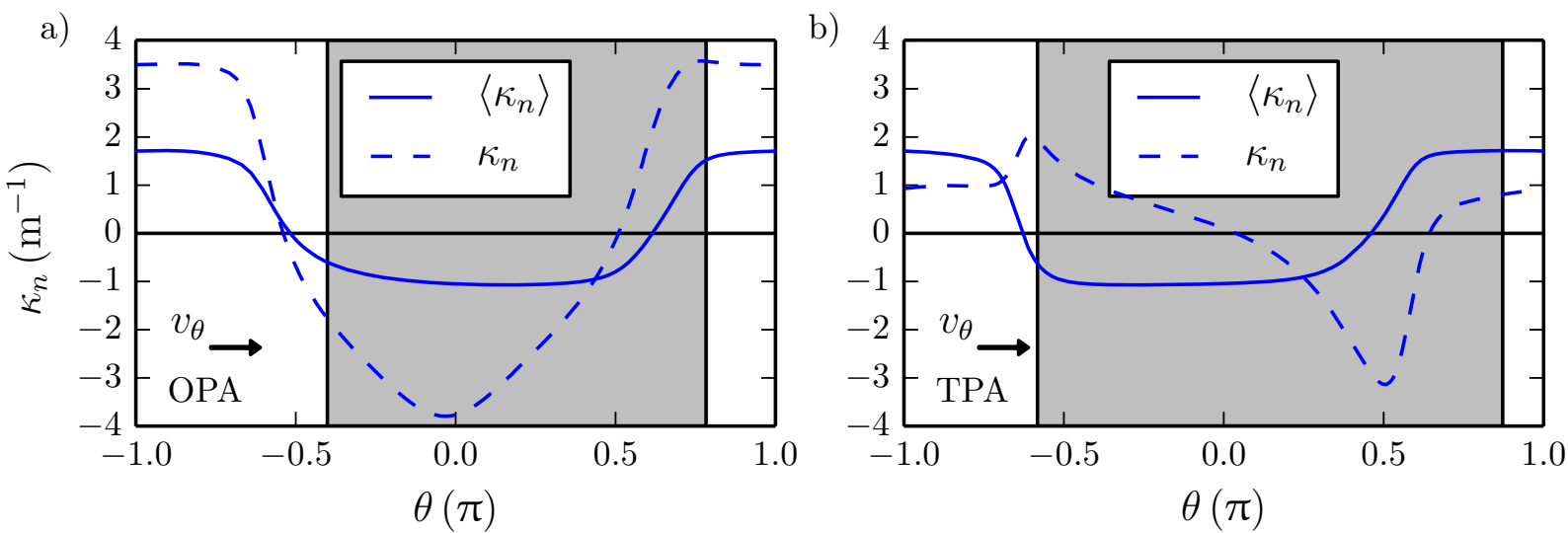

Figure 7. Local and averaged normal curvature $\left(\kappa_{\mathrm{n}}\right.$ and $\left.\left\langle\kappa_{\mathrm{n}}\right\rangle\right)$ as a function of the poloidal angle at the OPA (a) and TPA (b). The gray area marks the region where blobs are observed in discharge \#8355. The direction of the poloidal propagation of the blobs is indicated by the black arrow.
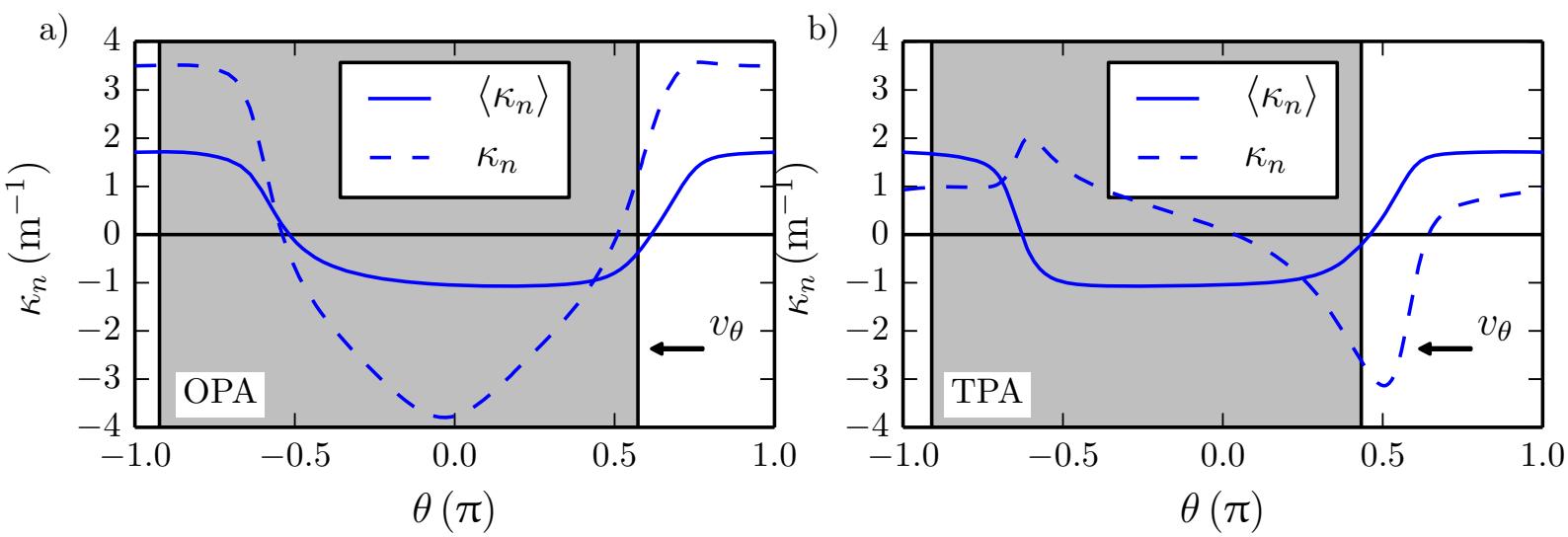

Figure 8. Same representation as in Fig. 7, but for the reversed field case (\#8352).

of the blobs is reversed. Hence, the entrance of the blob region is now located at large poloidal angles close to the zero crossing of $\left\langle\kappa_{\mathrm{n}}\right\rangle$ and the exit at negative poloidal angles in a region where $\left\langle\kappa_{\mathrm{n}}\right\rangle$ is positive again.

This shows that indeed blobs are generated in regions where $\left\langle\kappa_{\mathrm{n}}\right\rangle<0$ (in the following referred to as generation region, but due to their poloidal propagation they enter stable regions where $\left\langle\kappa_{\mathrm{n}}\right\rangle>0$. This is in agreement with the camera measurements discussed above, where a high cross correlation is found between a reference probe outside of the region of $\left\langle\kappa_{\mathrm{n}}\right\rangle<0$ and the image data showing a blob inside the region of $\left\langle\kappa_{\mathrm{n}}\right\rangle<0$ (Fig. 4 c)). Typical lifetimes of turbulent structures in the edge of TJ-K are of the order of $100 \mu \mathrm{s}$ [26], which is comparable to the time duration blobs are observed in the SOL. Hence, the difference between region I and III is probably simply that blobs decay in region III before they could reach region I.

The propagation of turbulent structures from unstable into stable regions is an example of turbulence spreading (i.e. the propagation of turbulent structures into regions, which themselves are stable against this kind of instability [27-29]).

In summary, blobs in the SOL of TJ-K are filaments elongated along the magnetic field lines, filling the space between the limiter disks. They are generated in regions of 
negative mean normal curvature $\left\langle\kappa_{\mathrm{n}}\right\rangle$. The locations of the limiter disks determine $\left\langle\kappa_{\mathrm{n}}\right\rangle$ at a specific location in the SOL and, hence, the limiters are breaking the stellarator symmetry of blob generation. A consequence of this symmetry breaking is that experiments with different measurement locations or limiter setups observe different regions of strong blob activity (e. g. at the outer midplane in Ref. [19] or in the bottom region in Ref. [13])

Due to the field alignment, a measurement of density fluctuations over a poloidal cross section of the SOL is sufficient to approximately reconstruct the whole filament. Using the conditionally averaged 2D data of density fluctuations associated with blobs from Ref. [13], such a 3D reconstruction has been performed as follows: In the 2D plane, the center of mass of the density structure associated with the blobs is determined. Then, the field line which crosses this center of mass is traced between the two limiter disks with a magnetic field-line code. The result is shown in Fig. 9.

The blob is visible as filament in the SOL and as the time progresses it propagates radially outward and poloidally into the ion diamagnetic drift direction. This gives a graphical summary of the blob structure and propagation in the stellarator TJ-K. The main difference to density perturbations arising due to drift-wave turbulence in the confined plasma (e.g. presented in Ref. [26]) is the missing poloidal and parallel mode structure, the poloidal propagation in the ion-diamagnetic direction, and the pronounced radial propagation.

\section{Blob induced transport}

In the last section it was shown that blobs in the stellarator TJ-K are generated in SOL regions with negative mean normal curvature, which agrees perfectly with the common picture of interchange-driven blobs. In the following it is studied to which extent these blobs contribute to the turbulent SOL transport in TJ-K.

Both the local and total turbulent transport $\Gamma$ can be measured by the poloidal probe arrays as described in Ref. [30]. In order to assess the contribution of blobs to the turbulent transport the following procedure is applied: Using the conditional averaging technique, the transport averaged over all events exceeding a specific density fluctuation threshold is determined (employed parameters: window size of 256 samples and threshold amplitude of $2 \sigma$ ). The cumulated time duration of all averaged events $T_{\mathrm{CA}}$ is recorded (number of trigger events multiplied by the chosen time interval length around the trigger events). The conditionally averaged transport is then compared to the transport averaged over the complement of the time trace $\left(\bar{T}_{\mathrm{CA}}\right)$, where no large amplitude events occur. The relative importance of blobs to the transport can then be estimated by

$$
f_{\Gamma, \text { blob }}=\frac{\langle\Gamma\rangle_{T_{\mathrm{CA}}}-\langle\Gamma\rangle_{\bar{T}_{\mathrm{CA}}}}{\langle\Gamma\rangle} \cdot \frac{T_{\mathrm{CA}}}{T_{\mathrm{CA}}+\bar{T}_{\mathrm{CA}}} .
$$

The first fraction describes the relative transport increase during a blob event: The conditionally averaged transport $\langle\Gamma\rangle_{T_{\mathrm{CA}}}$ during blob activity is reduced by the transport 

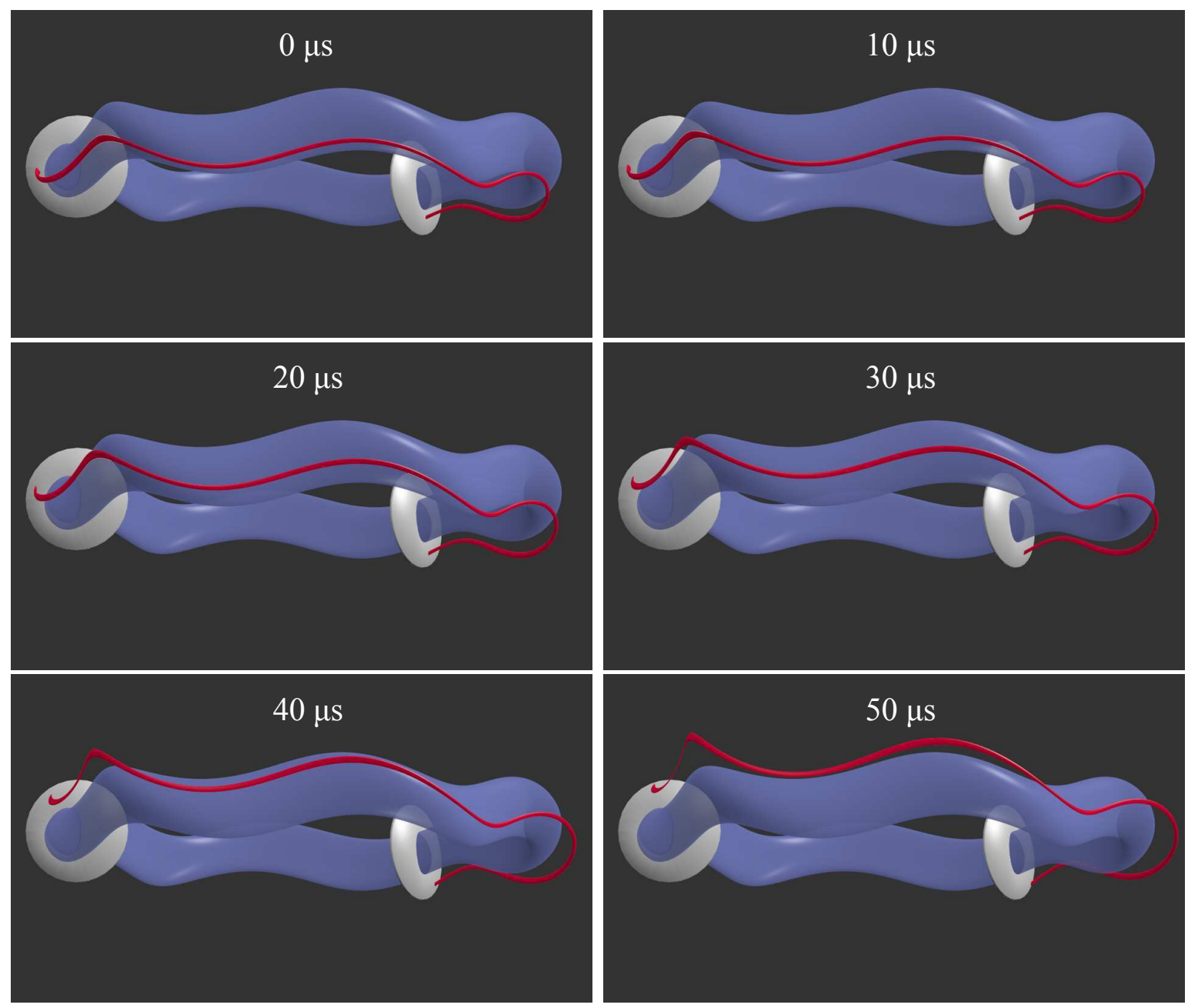

Figure 9. 3D reconstruction of a blob from $2 \mathrm{D}$ probe data. The confined plasma of TJ-K in the limiter configuration is shown in blue, the two limiter disks in gray, and the blob filament is shown in red. Depicted is the blob evolution in $10 \mu$ s steps.

averaged over time when no blobs are present $\langle\Gamma\rangle_{\bar{T}_{\mathrm{CA}}}$ (conservatively assuming that the ambient turbulent transport is not reduced in phases of blob activity) and the result is compared to the mean transport $\langle\Gamma\rangle$ averaged over the whole time trace. The second fraction represents how much of the time trace of duration $T=T_{\mathrm{CA}}+\bar{T}_{\mathrm{CA}}$ is covered by blob events and, hence, measures the relevance of blobs for the total turbulent transport.

In order to assess the contribution of blobs to the turbulent transport the camera was used to detect blobs originating from the generation region and conditionally average the corresponding transport as measured by the OPA. The result is illustrated in Fig. 10. For the conditional averaging a threshold value of two times the standard deviation $(\sigma)$ was used for light fluctuations at a reference pixel (indicated as blue dot in the Figure, refer to Ref. [13] for details on the image interpretation). The conditional average of the image data (left) shows a blob in the SOL. At the same time, the transport is increased very localized at poloidal angles around $\theta=[0,0.25 \pi]$ at port $\mathrm{O} 2$ (right). The maximum in the local transport at the OPA occurs at $\theta=0.07 \pi$. Figure 11 shows the 

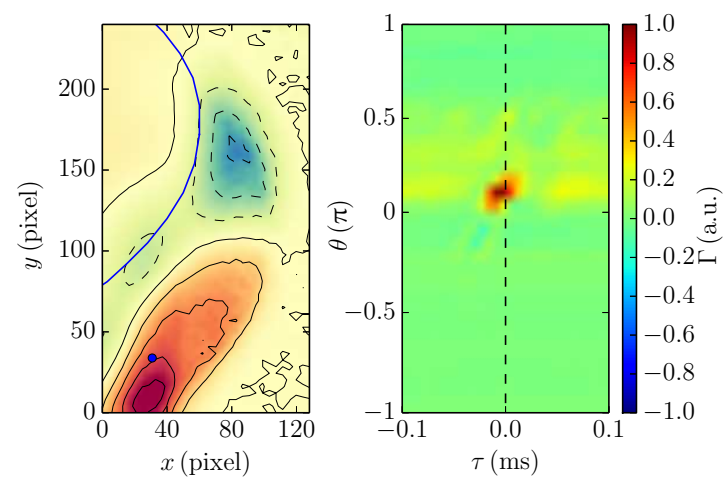

Figure 10. On the left, the conditional average of the normalized intensity fluctuations observed by the camera is shown (\#8354) at $\tau=0 \mu$ s showing a blob in the SOL. On the right, the conditional average of the local transport measured by the OPA is shown as a function of the time lag $\tau$ of the conditional average and the poloidal angle of the respective OPA probe. The blue dot in the camera image indicates the mapping along the field line of the OPA probe at $\theta=0.0$ where the transport maximum is observed and at the same time the pixel position of the reference signal for the conditional averaging.

conditionally averaged local transport $\Gamma(\theta=0.07 \pi)$ measured at that probe position. For a short time during the blob event the local transport increases by a factor of 9 .

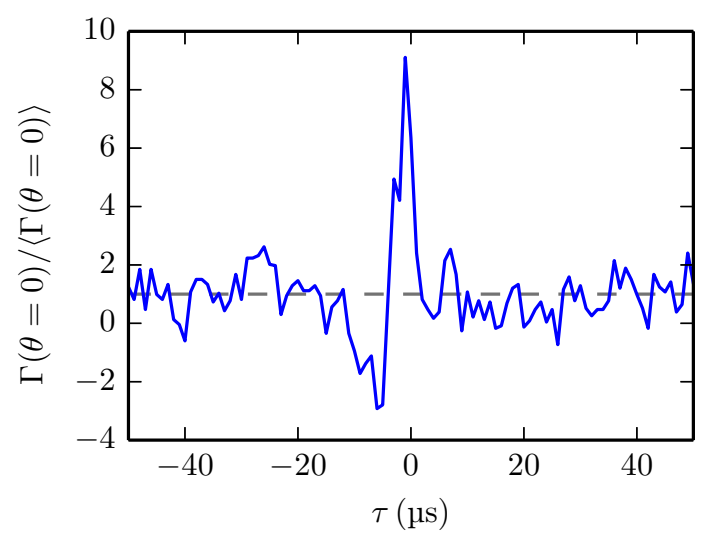

Figure 11. CA of the local transport at $\theta=0$ measured by the OPA normalized to its mean using the normalized intensity fluctuations from the camera data as reference signal (\#8354). The dashed line (gray) depicts the average transport level.

The relative importance over the whole time series estimated by Eq. (4) at $\theta=0.07 \pi$ is $95 \%$ for hydrogen and $53 \%$ for helium. In the generation region the local transport is clearly dominated by blobs.

The transport measured with the OPA is summed up over all probe positions $i$ in order to estimate the total (time dependant) transport $\Gamma \approx \Sigma_{i} \Gamma_{i} . \quad \Gamma$ is used as reference signal for a conditional averaging with a threshold value of two times the standard deviation. With this reference signal the conditional averages of $\Gamma_{i}, \Gamma$ itself, and the normalized intensity fluctuations recorded by the fast camera are determined. 
The result is shown in Fig. 12. A blob originating from the generation region is seen,
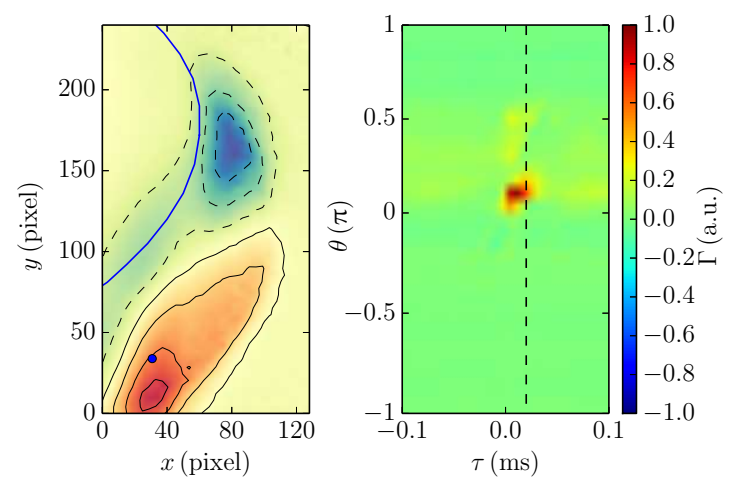

Figure 12. Same representation as in Fig. 10, but with the total transport $\Sigma_{i} \Gamma_{i}$ as reference for \#8354.

indicating that a significant fraction of peaks in the total transport correspond to blobs. On the right hand side of Fig. 12, the conditional average of the local transport is shown. It resembles the local transport profile already seen in Fig 10. Figure 13 shows the conditional average of $\Gamma$ with itself as reference signal. The total transport during a blob event increases by more than a factor of 4 . The fraction of blobby transport according to Eq. (4) is $23 \%$ for helium (\#8355) and $30 \%$ for hydrogen (\#8352). Hence, blobs contribute significantly to the total turbulent SOL transport in TJ-K and are observed as intermittent large-amplitude peaks in the transport. It is also clear that the majority of the turbulent transport, however, is caused by small-amplitude fluctuations about which these experiments give no information.

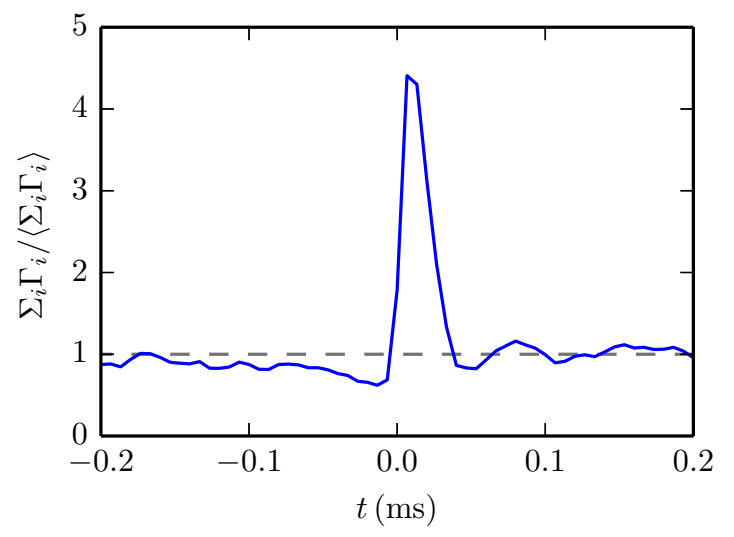

Figure 13. Conditional average of the relative total turbulent transport with itself as reference signal (\#8354). Blobs increase the transport temporarily by a factor of 4 .

\section{Summary and conclusion}

Experiments were conducted in the stellarator TJ-K to show that the basic picture of blob dynamics, despite the simplifications discussed in the introduction, is valid also 
in the complex magnetic geometry of a stellarator. By two poloidal probe arrays and a fast camera it was shown that blobs in TJ-K are field-aligned filaments in the SOL extended over the whole connection length between two limiters, which agrees with the common blob picture for sheath-limited blobs in simpler geometries.

The blobs are generated exclusively in regions of negative mean normal curvature (averaged along the field lines). The link between the relatively successfully described tokamaks and stellarators is the replacement of the $1 / R$ term by the mean normal curvature (averaged along the field line) $\left\langle\kappa_{\mathrm{n}}\right\rangle$ in the basic blob equations. Depending on the field geometry this can lead to a blob generation region not following the stellarator symmetry, as it is the case for the limiter setup of TJ-K deployed in the presented experiments. However, it was also observed that blobs are not restricted to this generation region since after their generation they propagate poloidally in interchange stable regions, which is a clear example of turbulence spreading.

A further result is that in agreement with similar observations from tokamaks [5, 31], the blobs in TJ-K contribute significantly to the turbulent transport in the SOL. Not only do blobs dominate the local turbulent transport in the generation region (in some experiments locally up to $95 \%$ ), but they also contribute significantly to the total turbulent transport in the SOL (around $30 \%$ ).

\section{References}

[1] Zweben S J and Gould R W 1985 Nucl. Fusion 25171

[2] Bleuel J, Endler M, Niedermeyer H, Schubert M, Thomsen H, and The W7-AS Team 2002 New J. Phys. 438

[3] Grulke O, Terry J L, Cziegler I, LaBombard B, and Garcia O E 2014 Nucl. Fusion 54043012

[4] Agostini M, Scarin P, Spizzo G, Vianello N, and Carraro L 2014 Plasma Phys. Controlled Fusion 56095016

[5] Boedo J A et al 2001 Phys. Plasmas 84826

[6] Terry J L, Zweben S J, Umansky M V, Cziegler I, Grulke O, LaBombard B, and Stotler D P 2009 J. Nucl. Mater. 390-391 339-342

[7] Fedorczak N, Manz P, Thakur S C, Xu M, Tynan G R, Xu G S, and Liu S C 2012 Phys. Plasmas 19122302

[8] Krasheninnikov S I 2001 Phys. Lett. A 283368

[9] Garcia O E, Bian N H, Naulin V, Nielsen A H, and Juul Rasmussen J 2005 Phys Plasmas 12090701

[10] Myra J R, D'Ippolito D A, Stotler D P, Zweben S J, LeBlanc B P, Menard J E, Maqueda R J, and Boedo J 2006 Phys. Plasmas 13092509

[11] D'Ippolito D A, Myra J R, and Zweben S J 2011 Phys. Plasmas 1860501

[12] Krasheninnikov S I, D’Ippolito D A, and Myra J R 2008 J. Plasma Phys. 74679 
[13] Fuchert G, Birkenmeier G, Nold B, Ramisch M, and Stroth U 2013 Plasma Phys. Control. Fusion 55125002

[14] D'Ippolito D A, Myra J R, and Krasheninnikov S I 2002 Phys. Plasmas 9222

[15] Theiler C, Furno I, Ricci P, Fasoli A, Labit B, Müller S H, and Plyushchev G 2009 Phys. Rev. Lett. 103065001

[16] Manz P, Carralero D, Birkenmeier G, Müller H W, Müller S H, Fuchert G, Scott B D, and Stroth U 2013 Phys. Plasmas 20102307

[17] Ramisch M, Birkenmeier G, Happel T, Köhn A, Mahdizadeh N, Manz P, Nold B, Wilcox R, Anderson D T, and Stroth U 2010 Contrib. Plasma Phys. 50 718-723

[18] Dewhurst J M, Hnat B, Ohno N, Dendy R O, Masuzaki S, Morisaki T, and Komori A 2008 Plasma Phys. Control. Fusion 50095013

[19] Happel T, Greiner F, Mahdizadeh N, Nold B, Ramisch M, and Stroth U 2009 Phys. Rev. Lett. 102255001

[20] Alonso J A et al 2006 Plasma Phys. Control. Fusion 48 B465-B473

[21] Simon P, Ramisch M, Beletskii A A, Dinklage A, Endler M, Marsen S, Nold B, Stroth U, Tamain P, and Wilcox R 2014 Plasma Phys. Control. Fusion 56095015

[22] Grulke O, Klinger T, Endler M, Piel A, and W7-AS Team 2001 Phys. Plasmas 8 5171

[23] Krause N, Lechte C, Stober J, Stroth U, Ascasibar E, Alonso J and Niedner S 2002 Rev. Sci. Instrum. 733474

[24] Stroth U, Greiner F, Lechte C, Mahdizadeh N, Rahbarnia K and Ramisch M 2004 Phys. Plasmas 112558

[25] Birkenmeier G, Ramisch M, Manz P, Nold B and Stroth U 2011 Phys. Rev. Lett. 107025001

[26] Birkenmeier G, Ramisch M, Fuchert G, Köhn A, Nold B, and Stroth U 2013 Plasma Phys. Control. Fusion 55015003

[27] Naulin V, Nielsen A H, and Juul Rasmussen J 2005 Phys. Plasmas 12122306

[28] Gürcan O D, Diamond P H, Hahm T S, and Lin Z 2005 Phys. Plasmas 12032303

[29] Manz P, Ribeiro T T, Scott B D, Birkenmeier G, Carralero D, Fuchert G, Müller S H, Müller H W, Stroth U, and Wolfrum E 2015 Phys. Plasmas 22022308

[30] Birkenmeier G, Ramisch M, Schmid B, and Stroth 2013 Phys. Rev. Lett. 110145004

[31] Carralero D, Birkenmeier G, Müller H W, Manz P, deMarne P, Müller S H, Reimold F, Stroth U, Wischmeier M, Wolfrum E, and the ASDEX Upgrade Team 2014 Nucl. Fusion $\mathbf{5 4} 123005$ 